\title{
Robert Langlands Awarded Abel Prize
}

The Norwegian Academy of Science and Letters has awarded the Abel Prize for 2018 to RoBERT P. LANGLANDS of the Institute for Advanced Study, Princeton, "for his visionary program connecting representation theory to number theory." The Abel Prize recognizes contributions of extraordinary depth and influence in the mathematical sciences and has been awarded annually since 2003. It carries a cash award of 6 million Norwegian krona (about US\$715,000).

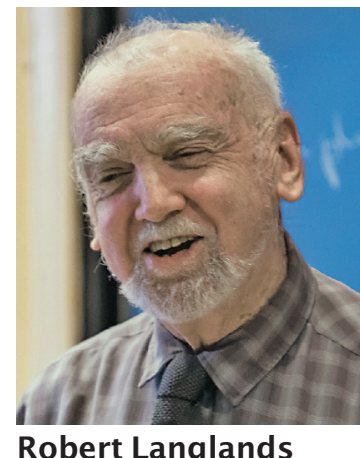

Robert Langlands

\section{Citation}

The Langlands Program predicts the existence of a tight web of connections between automorphic forms and Galois groups.

The great achievement of algebraic number theory in the first third of the twentieth century was class field theory. This theory is a vast generalization of Gauss's law of quadratic reciprocity. It provides an array of powerful tools for studying problems governed by abelian Galois groups. The non-abelian case turns out to be substantially deeper. Langlands, in a famous letter to André Weil in 1967, outlined a far-reaching program that revolutionized the understanding of this problem.

Langlands' recognition that one should relate representations of Galois groups to automorphic forms involves an unexpected and fundamental insight, now called Langlands functoriality. The key tenet of Langlands functoriality is that automorphic representations of a reductive group should be related, via $L$-functions, to Galois representations in a dual group.

Jacquet and Langlands were able to establish a first case of functoriality for GL(2), using the Selberg trace formula. Langlands' work on base change for GL(2) proved further cases of functoriality, which played a role in Wiles' proof of important cases of the Shimura-Taniyama-Weil conjecture.

The group GL(2) is the simplest example of a non-abelian reductive group. To proceed to the general case, Langlands saw the need for a stable trace formula, now established by Arthur. Together with Ngô's proof of the so-called Fundamental Lemma, conjectured by Langlands, this has led to the endoscopic classification of automor-

For permission to reprint this article, please contact: reprint-permission@ams.org.

DOI: http://dx.doi.org/10.1090/noti1692 phic representations of classical groups, in terms of those of general linear groups.

Functoriality dramatically unifies a number of important results, including the modularity of elliptic curves and the proof of the Sato-Tate conjecture. It also lends weight to many outstanding conjectures, such as the Ramanujan-Peterson and Selberg conjectures and the Hasse-Weil conjecture for zeta functions.

Functoriality for reductive groups over number fields remains out of reach, but great progress has been achieved by the work of many experts, including the Fields medalists Drinfel'd, Lafforgue, and Ngô, all inspired by the guiding light of the Langlands Program. New facets of the theory have evolved, such as the Langlands conjectures over local fields and function fields and the geometric Langlands Program. Langlands' ideas have elevated automorphic representations to a profound role in other areas of mathematics, far beyond the wildest dreams of early pioneers such as Weyl and Harish-Chandra.

\section{Biographical Sketch}

Following is a biography written by Alexander Bellos and published on the Wolf Foundation site (www. abelprize. no/c73016/binfi1/down1oad.php?tid=72984).

In January 1967, Robert Langlands, a thirty-year-old associate professor at Princeton, wrote a letter to the great French mathematician André Weil, age sixty, outlining some of his new mathematical insights. "If you are willing to read it as pure speculation I would appreciate that," he wrote. "If not-I am sure you have a waste basket handy." Langlands' modesty now reads like an almost comic piece of understatement. His seventeen-page letter introduced a theory that created a whole new way of thinking about mathematics: it suggested deep links between two areas, number theory and harmonic analysis, that had previously been considered unrelated.

In fact, so radical were his insights, and so rich the mechanisms he suggested to bridge these mathematical fields, that his letter began a project, the Langlands Program, that has enlisted hundreds of the world's best mathematicians over the last fifty years. No other project 
in modern mathematics has as wide a scope, has produced so many deep results, and has so many people working on it. As its depth and breadth have grown, the Langlands Program is frequently described as a grand unified theory of mathematics.

Robert Phelan Langlands was born in New Westminster, Greater Vancouver, Canada, in 1936. When he was nine, he moved to a small tourist town near the US border where his parents had a shop selling building supply materials. He had no intention of going to university until a teacher told him, in front of his classmates, that it would be a betrayal of his God-given talents.

Langlands enrolled at the University of British Columbia, aged sixteen. He completed his bachelor's degree in mathematics in 1957 and his master's degree a year later. He moved to Yale University for his doctorate, completing his PhD thesis, Semi-Groups and Representations of Lie Groups, in his first year there. In his second year he began to study the work of the Norwegian Atle Selberg, which later became central to his own research.

In 1960, Langlands joined Princeton University as an instructor, where he rubbed shoulders with Selberg, as well as André Weil and Harish-Chandra, all of whom were at the nearby Institute for Advanced Study. He was especially influenced by the work of Harish-Chandra on automorphic forms. Langlands was also learning other areas of mathematics, such as class field theory, an area he was nudged into by his colleague Salomon Bochner, who encouraged him to give a course in it. In 1962, Langlands was appointed a member in the Institute's School of Mathematics.

During the Christmas break of 1966, Langlands came up with the basic idea of "functoriality," a mechanism for linking ideas in number theory to those in automorphic forms. He bumped into Weil in a corridor in the beginning of January 1967 and began to explain his discovery. Weil suggested he write up his thoughts in a letter.

Langlands swiftly wrote the letter in longhand. Weil had the letter typed up, and it was widely circulated among mathematicians. Over the next few years, the letter provided many of them with a number of new, deep, and interesting problems, and, as more people joined the project to prove his conjectures, the enterprise became known as the Langlands Program. "There were some fine points that were right that rather surprise me to this day," Langlands later said about the letter. "There was evidence that these $L$-functions were good but that they would have these consequences for algebraic number theory was by no means certain."

Langlands spent the year 1967-1968 at the Middle East Technical University in Ankara. He speaks fluent Turkish. An enthusiastic learner of languages, he also speaks German and Russian.

Langlands returned to Yale, where he developed his twin ideas of functoriality and reciprocity and published them in "Problems in the Theory of Automorphic Forms" (1970). In 1972 he returned to Princeton as a professor at the Institute for Advanced Study, where he has been ever since.
Throughout the 1970s, Langlands continued to work on ideas within his program. In the mid-1980s, he turned his attention to percolation and conformal invariance, problems from theoretical physics. In recent years he has been looking back at ideas that he pioneered, such as one called "endoscopy".

Langlands' many honors include the first US National Academy of Sciences Award in Mathematics (1988); the Cole Prize in Number Theory (1982, with Barry Mazur); the Wolf Prize (1995-1996, with Andrew Wiles); the Steele Prize for Seminal Contribution to Research (2005); the Nemmers Prize (2006); and the Shaw Prize (2007, with Richard Taylor). He was a member of the inaugural class of AMS Fellows (2012).

AMS President Kenneth A. Ribet said, "It is my great pleasure to congratulate Professor Robert P. Langlands, winner of the 2018 Abel Prize. Robert Langlands is one of the most distinguished mathematicians alive today and a towering figure in the history of modern mathematics. His insights, which grew out of penetrating technical work early in his career, have transformed and enriched both number theory and representation theory. The deep relations between the two subjects that he predicted and probed have guided the work of countless mathematicians over the last 50 years."

Read more about Langlands' life and work, including "A Glimpse of the Laureate's Work" by Alex Bellos and "17 Handwritten Pages That Shaped a Whole Area of Mathematical Research" and "From Quadratic Reciprocity to Langlands' Program" by Arne B. Sletsjøe at www. abe1prize. no/c73016/seksjon/vis.htm1?tid=73017\&strukt_ tid=73016.

\section{About the Prize}

The Niels Henrik Abel Memorial Fund was established in 2002 to award the Abel Prize for outstanding scientific work in the field of mathematics. The prize is awarded by the Norwegian Academy of Science and Letters, and the choice of Abel Laureate is based on the recommendation of the Abel Committee, which consists of five internationally recognized research scientists in the field of mathematics. The Committee is appointed for a period of two years. The members of the current committee are:

- John Rognes, University of Oslo (Chair)

- Marie-France Vignéras, Institut de Mathématiques de Jussieu

- Ben J. Green, University of Oxford

- Irene Fonseca, Mellon College of Science

- Sun-Yung Alice Chang, Princeton University Previous recipients of the Abel Prize are:

- Jean-Pierre Serre (2003)

- Michael Atiyah and I. M. Singer (2004)

- Peter Lax (2005)

- Lennart Carleson (2006)

- S. R. S. Varadhan (2007)

- John G. Thompson and Jacques Tits (2008)

- Mikhail L. Gromov (2009)

- John Tate (2010)

- John Milnor (2011) 


\section{AMS COMMUNICATION}

- Endre Szemerédi (2012)

- Pierre Deligne (2013)

- Yakov Sinai (2014)

- John F. Nash Jr. and Louis Nirenberg (2015)

- Andrew J. Wiles (2016)

- Yves Meyer (2017)

-From announcements of the Norwegian Academy of Science and Letters

\section{Photo Credit}

Photo of Robert Langlands courtesy of Dan Komoda/ Institute for Advanced Study.

See the WHAT IS? article on The Langlands Program in this issue, page 663.

\section{Author} Resource Center
The American Mathematical Society welcomes you to use the information and tools provided in our online Author Resource Center to prepare your work for publication. The Center is available to assist you with successfully writing, editing, illustrating, and publishing your mathematical works.

\section{Visit www.ams.org/authors to start utilizing this great resource!}

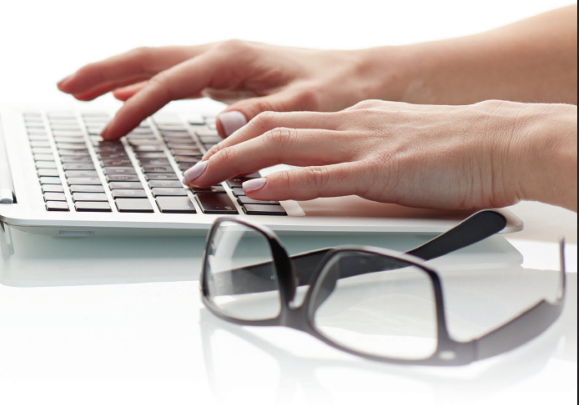

AMS 